\title{
Análisis del funcionamiento económico productivo de los sistemas de producción cárnica bovina en la Amazonía Ecuatoriana
}

\author{
Ríos-Núñez, S. 1,2,@y Benítez-Jiménez, D. ${ }^{2}$
}

'CEDER. Universidad de Los Lagos. Chile.

2Departamento de Ingenieria Agropecuaria. Universidad Estatal Amazónica. Ecuador.

Palabras ClaVe adicIONALES

Caracterización.

Ganadería bovina cárnica.

Amazonía Ecuatoriana.

\section{RESUMEN}

En la presente investigación se analiza la situación del sector ganadero bovino cárnico en la provincia de Pastaza (todos los estudios existentes se refieren a la ganadería de las provincias ganaderas por excelencia ubicadas en las provincias de Napo, Morona Santiago y Zamora Chinchipe), Amazonía Ecuatoriana. La participación de esta provincia en el escenario amazónico es reducida $(5,2 \%$ de la ganadería bovina total), sin embargo, en los últimos diez años este territorio ha tenido un incremento del 69,2\% de la superficie de pastos unido a una importante reducción del área de bosques. Se presentan y analizan los resultados descriptivos de 86 encuestas realizadas a explotaciones ganaderas donde se profundiza en sus principales dinámicas de funcionamiento identificando las fortalezas y debilidades que presenta actualmente la actividad. Las explotaciones encuestadas se caracterizan por su marcado carácter familiar, con importantes deficiencias en alimentación animal por el sistema de pastoreo y pastos utilizados, resultados económicos negativos y bajo relevo generacional por las condiciones extremas donde se realiza la ganadería. Esta actividad se desarrolla en un ecosistema frágil, con condiciones edafoclimáticas particulares que condicionan su desempeño económico-productivo. En este contexto, se hace necesario implementar un sistema de gestión de la ganadería que garantice el desarrollo de una actividad pecuaria que mejore su eficiencia económica- productiva y que al mismo tiempo reduzca la frontera ganadera mitigando el impacto ambiental que genera sobre el ecosistema.

Analysis of productive economic performance of bovine production systems in the Ecuadorian Amazonia

\section{SUMMARY}

\section{ADDITIONAL KEYWORDS}

Characterization.

Beef cattle livestock.

Ecuadorian Amazonia.

INFORMACIÓN

Cronología del artíiculo.

Recibido/Received: 9.4.2015

Aceptado/Accepted: 9.10.2015

On-line: 10.12 .2015

Correspondencia a los autores/Contact e-mail:

sandra.rios@ulagos.cl

\section{INTRODUCCIÓN}

La ganadería bovina en la Amazonía Ecuatoriana es una actividad introducida en la década de los sesenta como parte de la última etapa de colonización que tuvo lugar en el país. En este periodo se expandió la frontera agrícola y se desarrollaron importantes vías para facilitar la exploración y explotación petrolera (Barsky et al., 1982) que tuvo lugar en el país. Una de las principales actividades agropecuarias que se fomentó en ese momento fue la ganadería, por tanto, la sustitución de bosques por pastos para el ganado fue una práctica habitual. Esta errada política estatal no 
consideró los umbrales de producción eco-sistémicos del territorio Amazónico lo que ha provocado una serie de importantes impactos negativos a nivel económico y socio-ambiental (Wunder, 2000; Ehrhart, 2000; Sierra y Stalling, 1998; Pichon, 1993; Rudel y Horowitz, 1993 y Descola, 1984). Sólo el 17,5\% del territorio tiene aptitud para producción agropecuaria (Nieto y Caicedo, 2012).

En la Amazonía Ecuatoriana el 53\% del uso del suelo corresponde a bosques y montes, el $40 \%$ a pastos (naturales y cultivados) y sólo el 5\% a cultivos permanentes y transitorios (INEC, 2013). Entre 2000-2011 se produjo una importante reducción de la superficie de bosques y montes $(-16,1 \%)$, cultivos permanentes $(-24,2 \%)$ y transitorios $(-47,4 \%)$ y el aumento de la superficie de pastos cultivados $(11,2 \%)$ y naturales (332,6\%) (INEC, 2013). La actividad ganadera está progresivamente creciendo en este frágil territorio y, por tanto, es relevante analizar los mecanismos de funcionamiento que tienen estos sistemas de manejo. En términos absolutos, la superficie de pastos aumentó en 168 mil hectáreas, lo que refleja un importante avance de la frontera ganadera. La evolución del suelo a nivel provincial presenta dinámicas similares al conjunto de la Amazonía Ecuatoriana. La mayor variación ha tenido lugar en la provincia de Pastaza. Entre 2000-2011 los pastos han aumentado en 116 mil hectáreas representando el 69,2\% de todo el crecimiento de esta tipología de uso agrario en el territorio amazónico.

La provincia de Pastaza es una de las seis provincias de la Región Amazónica Ecuatoriana (RAE), tiene una superficie de $29773 \mathrm{~km}^{2}$ (figura 1) lo que equivale al $12 \%$ del país y al 25,5\% de la RAE. En este territorio solo algunos cultivos pueden adaptarse fácilmente a las condiciones climáticas extremas y a la pobre vocación agrícola de los suelos (Bravo, 2014; Nieto y Caicedo, 2012; Caballero y Anzules, 1994 y Bustamante et al., 1993). El 51,1\% del suelo está dedicado a bosques y montes, el $40,9 \%$ a pastos (naturales y cultivados) y sólo el 2,6\% a cultivos permanentes y transitorios (INEC, 2013). El 85\% del suelo con uso agropecuario se utiliza en sistemas ganaderos, superficie que crece a expensas de las áreas de bosques existentes en este ecosistema. En Pastaza existen 1663 explotaciones de las cuales $42,6 \%$ corresponden a lecherías doble propósito y el 57,4\% a cría-ceba (Vargas et al., 2014). Las condiciones edafoclimáticas del territorio estudiado condicionan la actividad ganadera imprimiéndole su carácter extremo, se observan valores medios en precipitaciones anuales de $4500 \mathrm{~mm} /$ año, temperaturas de 22 grados centígrados, humedad relativa de $70 \%$ y una altura de 900 msnm. Además el 30\% de la superficie no es apta para el pastoreo por la existencia de pendientes pronunciadas (sobre el 30\%) (Vargas et al., 2014, Benítez et al., 2010 y Murgueito e Ibrahim, 2004). El tipo de suelo predominante es el Inceptisol (78,6\% de la superficie), poco apto para el cultivo por abundancia de minerales como el aluminio. La vegetación es propia del bosque lluvioso tropical con un bajo nivel y retención de nutrientes lo que problemático cualquier cambio en el uso del suelo especialmente porque rompe con el ciclo de nutrientes auto-sostenible propio de la selva amazónica.

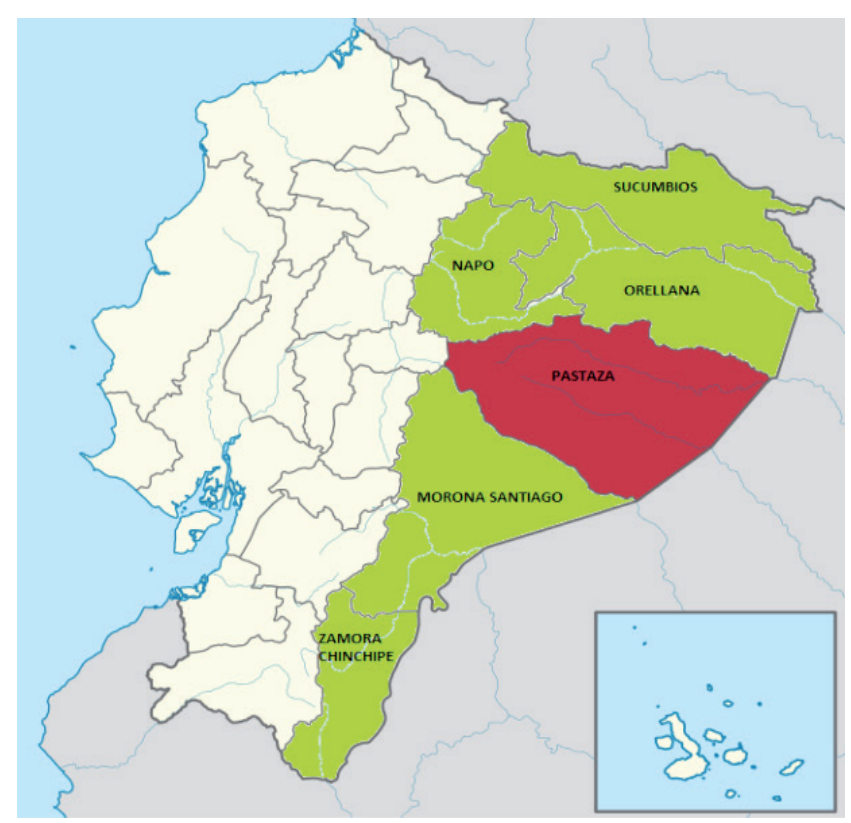

Figura 1. Amazonía Ecuatoriana y Provincia de Pastaza donde se realizaron las encuestas (Ecuadorian Amazonia and Pastaza Province where the surveys were conducted).

En este escenario a la ganadería se le considera como una de las actividades agropecuarias que causa mayor impacto negativo sobre el entorno amazónico asociándose a la deforestación, compactación de tierras por el pisoteo, erosión, perdida de la fertilidad de los suelos, reducción de la biodiversidad y contaminación de las corrientes de agua (INIAP, 2010 y Murgueitio e Ibrahim, 2004).

Actualmente la ganadería en la Amazonía Ecuatoriana es una actividad que se caracteriza por importantes deficiencias entre las que se destacan problemas productivos relacionados con el manejo de pastos para la alimentación del rebaño, manejo reproductivo y sanidad animal. Además los sistemas ganaderos tienen importantes problemas económicos tanto por la elevada dependencia de piensos industriales como por erradas estrategias comerciales.

En la medida que se realice una reestructuración de los sistemas ganaderos a nivel económico-productivo y de ordenamiento territorial que respete los umbrales de producción de un sistema frágil como es la Amazonía Ecuatoriana se podrá frenar el avance indiscriminado de la frontera ganadera en el territorio. Para llevar a cabo lo anterior, es necesario realizar un estudio del funcionamiento de estos sistemas ganaderos en cada provincia. Todos los estudios existentes se refieren a la ganadería de las provincias ganaderas por excelencia ubicadas en las provincias de Napo, Morona Santiago y Zamora Chinchipe. El objetivo de esta investigación es realizar un diagnóstico de la realidad económico-productiva de la ganadería bovina cárnica en la provincia de Pastaza (actualmente inexistente) con la finalidad de conocer sus dinámicas de funcionamiento e informar de sus posibilidades de mejora.

\section{MATERIAL Y MÉTODOS}

Se realizaron 86 encuestas a nivel de explotación en la provincia de Pastaza a ganaderos bovinos cárnicos 
Tabla I. Variables utilizadas en el análisis económico productivo (Variables used in the productive economic analysis).

\begin{tabular}{ll}
\hline Variables cuantitativas & Variables cualitativas \\
\hline Tamaño del rebaño (cabezas) & Relevo generacional \\
Mano de obra (UTA) & Razas existentes \\
Edad del titular (años) & Tipo de pastoreo \\
Años de experiencia en ganadería (años) & Alimentación animal \\
Prolificidad (terneros/vaca) & Método reproductivo \\
Edad destete (meses) & Manejo del ternero \\
Productividad cárnica (kilos/há) & Sanidad animal \\
Ingresos por UGM (Dolares/UGM) & Bienestar animal \\
Ingresos provenientes de la ganadería (\%) & \\
Principal coste fijo (\%) & \\
Principal coste variable (\%) & \\
Costes con MOF por UGM (Dólares/UGM) & \\
Costos sin MOF por UGM (Dólares/UGM) & \\
\hline
\end{tabular}

en 2014 (julio-noviembre) mediante muestreo aleatorio estratificado proporcional (figura 1). Las encuestas aplicadas suponen el $9 \%$ de la población estudiada y se realizaron especialmente para esta investigación.

Se ha considerado dos estratos, el del tamaño del rebaño (1-20 cabezas; 11-20; 21-60 y más de 60) y la orientación productiva (cría y ceba).

El censo utilizado ha sido el de control sanitario generado realizado por la Agencia Ecuatoriana de Aseguramiento de Calidad del Agro (AGROCALIDAD) de la provincia de Pastaza para el 2013.

Los datos recogidos en la encuesta son los relacionados con tamaño del rebaño, mano de obra, razas, producciones, manejo del rebaño y resultados económicos (tabla I), variables que en estudios similares han tenido un significativo nivel descriptivo a la hora de caracterizar el funcionamiento de los sistemas ganaderos bovinos en ecosistemas amazónicos ecuatorianos (Meunier, 2007 y Grijalva et al., 2004). Esta investigación es exploratoria-descriptiva, se realizaron análisis cuantitativo utilizando medidas de centralización y dispersión y análisis cualitativo a través de distribución de frecuencias. El programa estadístico utilizado ha sido el SPSS versión 22.0.

En cuanto a la obtención del desempeño económico de las explotaciones se ha cuantificado monetariamente la mano de obra familiar (MOF) al salario de mercado de la mano de obra asalariada y se ha realizado el análisis económico con y sin MOF para valorar la importancia que ésta tiene en los sistemas de manejo ganadero en la provincia.

\section{RESULTADOS}

Los sistemas de cría estudiados (62) tienen un manejo extensivo, la alimentación se basa en el pastoreo y en menor medida en la suplementación de concentrado. Los animales se venden al destete entre 12-14 meses. Los sistemas de ceba (24) cuentan con un manejo semi-intensivo, la alimentación base es el pastoreo y adicionalmente se suministra diariamente concentrado en los pastizales. Los animales se venden entre 30-40 meses. En ambos sistemas el pastoreo es al sogueo (sistema de pastoreo que amarra a los animales con una soga a un área determinada de pasto donde permanecen confinados) con un bajo aporte de nutrientes y la comercialización se realiza a través de intermediarios. A continuación se describe en detalle cada sistema de manejo.

\section{TAMAÑO DEL REBAÑO}

En cuanto al tamaño del rebaño, existen comportamientos distintos en cuanto a la orientación productiva cría o ceba. En el caso de la primera, la media es de $23,5 \pm 18,8$ y en el de la segunda, la media es menor $14,3 \pm 10,4$. El tamaño medio del rebaño agrupado según estratos presenta una variabilidad menor reflejada en sus variaciones típicas. Con todo, las ganaderías de cría tienen una mayor dimensión que las de ceba. Los primeros manejan rebaños de entre 11-20 cabezas $(46,8 \%)$ y los segundos manejan en promedio entre 1-10 cabezas (45,8\%) (tabla II).

\section{MANEJO DEL REBAÑO}

\section{AlimeNTACIÓN ANIMAL}

El principal pasto que manejan los ganaderos, tanto de cría $(100 \%)$ como de ceba $(79,2 \%)$, es la gramínea Axonopus scoparius (gramalote), pasto tropical perenne que produce estolones robustos. Su bajo contenido de materia seca por unidad de peso fresco digerida limita el crecimiento del ganado (Leonard, Andino y Uvidia, 2014). El gramalote tiene un ciclo largo de crecimiento (9-10 meses).

La utilización de pienso para los ganaderos encuestados oscila entre $40,3 \%$ para el caso de la cría y el $33,3 \%$ para los de ceba. Por tanto, sobre el $60 \%$ de los ganaderos utiliza el pastoreo del monte como único recurso de alimentación animal complementándola con fuentes energéticas como la caña de azúcar (tabla III).

Tabla II. Estructura de los rebaños (Herd structure).

\begin{tabular}{|c|c|c|c|c|c|c|c|c|}
\hline \multirow{2}{*}{ Estratos (cabezas) } & \multicolumn{4}{|c|}{ Grupo I cría $(n=62)$} & \multicolumn{4}{|c|}{ Grupo II ceba $(n=24)$} \\
\hline & Ganaderías & Media & DT & Intervalos & Ganaderías & Media & DT & Intervalos \\
\hline $1-10$ & $11(17,7)$ & 8,4 & 1,96 & $4-10$ & $11(45,8)$ & 6,7 & 6,0 & $1-22$ \\
\hline $11-20$ & $29(46,8)$ & 14,8 & 2,46 & $11-20$ & $9(37,5)$ & 15,7 & 3,6 & $11-20$ \\
\hline $21-60$ & $17(27,4)$ & 33,5 & 11,3 & $21-59$ & $4(16,7)$ & 32,3 & 5,2 & $29-40$ \\
\hline Más de 60 & $5(8,1)$ & 72,8 & 12,7 & $61-91$ & 0 & - & - & - \\
\hline Total & $62(100)$ & 23,5 & 18,8 & 4-91 & $24(100)$ & 14,3 & 10,4 & $1-40$ \\
\hline
\end{tabular}


Tabla III. Variables cualitativas del manejo ganadero (Livestock handling qualitative variables).

\begin{tabular}{lcccrrrr}
\hline \multicolumn{7}{c}{ Grupo I cría $(n=62)$} & \multicolumn{5}{c}{ Grupo II ceba $(n=24)$} \\
\hline & F & $\%$ & A & F & $\%$ & A \\
\hline Relevo generacional & & & & & & \\
\hline Sí & 44 & 71 & 71 & 18 & 75 & 75 \\
No & 18 & 29 & 100 & 6 & 25 & 100 \\
\hline Asociación agraria & & & & & & \\
\hline Sí & 25 & 40,3 & 40,3 & 9 & 37,5 & 37,5 \\
No & 37 & 59,7 & 100 & 15 & 62,5 & 100 \\
\hline
\end{tabular}

Razas existentes

\begin{tabular}{lrrrrrc}
\hline Mestiza sin definir & 27 & 43,6 & 43,6 & 13 & 59,1 & 59,1 \\
Mestiza Charolés & 16 & 25,8 & 69,4 & 3 & 13,6 & 72,7 \\
Mestiza Holstein & 10 & 16,1 & 85,5 & 3 & 13,6 & 86,4 \\
Mestiza Parda Alpina & 9 & 14,5 & 100 & 3 & 13,6 & 100 \\
\hline Tipo de mano de obra & & & & & & \\
\hline Sólo familiar & 56 & 90,3 & 90,3 & 22 & 91,7 & 91,7 \\
Asalariada & 6 & 9,7 & 100 & 2 & 8,3 & 100 \\
\hline Tipo de pastoreo & & & & & & \\
\hline Sogueo & 62 & 100 & 100 & 20 & 83,3 & 83,3 \\
Rotacional & & & & 4 & 16,7 & 100 \\
Racional & & & & & & \\
Continuo & & & & & & \\
\hline
\end{tabular}

\begin{tabular}{lrrrrrc}
\hline Pastura predominante & & & & & & \\
\hline Gramalote & 62 & 100 & 100 & 19 & 79,2 & 79,2 \\
$\begin{array}{l}\text { Rastreros } \\
\text { Leguminosas }\end{array}$ & & & & 1 & 4,2 & 83,3 \\
Erectos & & & & 4 & 16,7 & 100 \\
\hline Utilización de pienso & & & & & & \\
\hline Sí & 25 & 40,3 & 40,3 & 8 & 33,3 & 33,3 \\
No & 37 & 59,7 & 100 & 16 & 66,7 & 100 \\
\hline Método reproductivo & & & & & & \\
\hline Monta natural & 39 & 62,9 & 62,9 & 11 & 45,8 & 45,8 \\
Inseminación artificial & 16 & 25,8 & 88,7 & 1 & 4,2 & 50 \\
Ambas & 7 & 11,3 & 100 & & & 50 \\
No utiliza & & & & 12 & 50 & 100 \\
\hline
\end{tabular}

Sistema de crianza del ternero

\begin{tabular}{lrrr}
\hline Natural & 40 & 64,5 & 64,5 \\
Artificial & 10 & 16,1 & 80,6 \\
$\begin{array}{l}\text { Amamantamiento } \\
\text { restringido }\end{array}$ & 12 & 19,4 & 100 \\
\hline
\end{tabular}

Sanidad animal

\begin{tabular}{lrrrrrr}
\hline \multicolumn{7}{l}{ Asistencia veterinaria permanente } \\
Sí & 7 & 11,3 & 11,3 & 6 & 25 & 25 \\
No & 55 & 88,7 & 100 & 18 & 75 & 100
\end{tabular}

Programa sanitario de prevención

\begin{tabular}{lllllll}
\hline Sí & 62 & 100 & 100 & 24 & 100 & 100 \\
No & & & & & & \\
\hline Registro reproductivo & & & & & & \\
\hline Sí & 62 & 100 & 100 & 24 & 100 & 100 \\
No & & &
\end{tabular}

Grupo I cría ( $n=62)$ Grupo II ceba $(n=24)$

$\begin{array}{llllll}\mathrm{F} & \% & \mathrm{~A} & \mathrm{~F} & \% & \mathrm{~A}\end{array}$

Bienestar animal

Disponibilidad de agua para el ganado

\begin{tabular}{|c|c|c|c|c|c|c|}
\hline Sí & 4 & 6,5 & 6,5 & 6 & 25 & 25 \\
\hline No & 58 & 93,5 & 100 & 18 & 75 & 100 \\
\hline \multicolumn{7}{|c|}{ Pendiente promedio área de pastoreo } \\
\hline Sobre $30 \%$ & 33 & 53,2 & 53,2 & 12 & 50 & 50 \\
\hline Menor al $30 \%$ & 29 & 46,8 & 100 & 12 & 50 & 100 \\
\hline
\end{tabular}

$F=$ Frecuencia; $A=$ Acumulado.

El principal método de pastoreo utilizado en la provincia es el sogueo para que Axonopus scoparius resista el pisoteo del ganado. Esta técnica consiste en que los animales son amarrados con una soga de aproximadamente 6-8 metros donde permanecen confinados. Éstos se movilizan al pasto fresco dos veces al día. Esta práctica permite optimizar la utilización del pasto al reducir los rechazos y el sobrepastoreo. Una vez que los animales consumen todo el forraje son cambiados de lugar. El ganado vuelve a las áreas consumidas a los 9-10 meses siguientes, donde esta gramínea está en condiciones de ser consumida nuevamente.

Los métodos reproductivos utilizados son principalmente monta natural en ambas orientaciones productivas. No existe estacionalidad reproductiva, los ganaderos no planifican la monta por grupos. La monta artificial se realiza a través de programas del gobierno local que fomentan el mejoramiento genético de la cabaña ganadera. En estos programas se brinda a los ganaderos asistencia técnica e insumos para realizar la inseminación artificial. No obstante, el déficit de técnicos, el difícil acceso a las explotaciones para asistir en tiempo de celo al ganado y la selección genética inadecuada al territorio no ha permitido mejorar los indicadores reproductivos del rebaño (tabla III).

En el caso de la cría, la crianza del ternero se realiza mayoritariamente de manera natural $(64,5 \%)$. La proli-

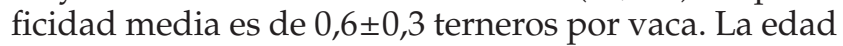
media del destete es de 22,5 \pm semanas (tabla III).

La sanidad animal en los ganaderos encuestados presenta importantes deficiencias. El 88,7\% de las ganaderías de cría y el $75 \%$ de las de ceba no cuentan con la asistencia de un médico veterinario de manera permanente. El 100\% de ellas no cuenta con un programa sanitario de prevención de enfermedades (tabla III).

Con respecto al bienestar animal, se utilizaron dos variables de análisis. Primero, la disponibilidad de agua que en todas las ganaderías es deficiente. El 93,5\% de los ganaderos de cría y el 75\% de los de ceba no brindan agua a sus animales. Segundo, las fuertes pendientes donde se realiza la ganadería provocan importantes problemas para el bienestar de los animales que sumado al pastoreo bajo la técnica del sogueo genera mortalidades de la cabaña ganadera. En torno al $50 \%$ de los ganaderos maneja su rebaño en pendientes en promedio sobre el 30\% (tabla III). 


\section{RAZAS}

La estructura racial de los rebaños se caracteriza por cruces mestizos sin tipos raciales definidos (43,6\% para el caso del ganado de cría y 59,1\% para el ganado de ceba). En el caso de la cría están presente además los cruces de Mestizos Charolés (25,8\%), Holstein (16,1\%) y Parda Alpina (14,5\%). En el caso de la ceba están presentes los mismos cruces mestizos con igual participación (13,6\% respectivamente) (tabla III).

\section{PRODUCCIONES Y COMERCIALIZACIÓN}

En cuanto al grado de asociatividad, los ganaderos de cría presentan un nivel de asociación mayor (40,3\%) con respecto a los ganaderos de ceba $(37,5 \%)$ (tabla III). No obstante, el poder de negociación de las organizaciones de ambas orientaciones productivas es bajo.

En el caso de la cría, el 100\% de los ganaderos encuestados comercializa bajo la modalidad de peso vivo a través de dos modalidades indistintamente. Primero, realizan un acuerdo de compra-venta entre ganaderos donde se transa el ganado en pie y se movilizan los animales entre las explotaciones de los ganaderos. Segundo, a través de la Feria de Comercialización de Ganado de Puyo, administrada por la Asociación de Ganaderos de Pastaza, ASOGAP. En esta feria se reúnen los ganaderos a transar ganado vivo. En el caso de la ceba, el $100 \%$ de los ganaderos encuestados comercializa su ganado bajo peso vivo utilizando tres canales de comercialización. Primero, a través de lo que se llama los arreglos en finca, acuerdo de compra-venta entre ganaderos y carnicerías (tercenistas) donde se transa el ganado en pie. Éste último realiza una oferta económica al ganadero por el o los animales que quiere adquirir. El sistema de fijación de precios es informal y se basa en la estimación del peso a ojo del peso del animal, donde el poder de negociación lo ejerce en su totalidad el tercenista. Posteriormente el ganado se lleva al Camal Municipal para sacrificio, el coste del transporte es asumido por el ganadero. Segundo, a través de lo que se llama los arreglos en el Camal, acuerdo de compra-venta entre ganaderos y tercenistas donde se transa el ganado en cuartos. El sistema de fijación de precios es al peso donde el tercenista paga los cuatro cuartos. Tercero, comercialización de ganado en finca, acuerdo de compra-venta entre ganaderos donde se transa el ganado en pie y se movilizan los animales entre las explotaciones de los ganaderos. Cuarto, a través de la Feria de Comercialización de Ganado de Puyo, administrada por la Asociación de Ganaderos de Pastaza, ASOGAP. En esta feria se reúnen los ganaderos a transar ganado vivo.

\section{MANO DE OBRA}

La media de la mano de obra por explotación presenta una realidad similar para ambas orientaciones productivas estudiadas. Para el caso de la cría, se utiliza en promedio 0,28 UTA (rango 0,1-0,6). Sólo el 9,7\% de los encuestados contrata mano de obra asalariada y es en su totalidad temporal. Para el caso de la ceba, los ganaderos utilizan en promedio 0,3 UTA (rango 0,11,3) con una variabilidad mayor. Sólo el 8,3\% utiliza mano de obra asalariada temporal. La importancia de la mano de obra familiar, por tanto, deja de manifiesto la característica de estos sistemas ganaderos en la Amazonía Ecuatoriana (tabla III y IV).

Una de las labores que requiere mayor participación de la mano de obra es el sogueo donde el ganadero cambia sus animales de lugar de pastoreo. Esto requiere que él se dirija al pastizal dos veces al día (por la maña-

Tabla IV. Variables cuantitativas del manejo ganadero (Livestock handling quantitative variables).

\begin{tabular}{|c|c|c|c|c|c|c|}
\hline & \multicolumn{3}{|c|}{ Grupo I cría $(n=62)$} & \multicolumn{3}{|c|}{ Grupo II ceba $(n=24)$} \\
\hline & $\mathrm{N}$ & Mínimo-Máximo & Media \pm DT & $\mathrm{N}$ & Mínimo-Máximo & Media $\pm \mathrm{DT}$ \\
\hline \multicolumn{7}{|l|}{ Mano de Obra } \\
\hline UTA total & 62 & $0,1-0,6$ & $0,28 \pm 0,09$ & 24 & $0,1-1,3$ & $0,3 \pm 0,2$ \\
\hline UTA familiar & 62 & $0,05-0,4$ & $0,26 \pm 0,07$ & 24 & $0,05-0,4$ & $0,26 \pm 0,96$ \\
\hline UTA asalariada & 62 & $0-0,2$ & $0,02 \pm 0,04$ & 24 & $0-1,1$ & $0,5 \pm 0,2$ \\
\hline Edad del titular & 62 & $21-90$ & $54,3 \pm 13,8$ & 24 & $29-92$ & $50,2 \pm 15,5$ \\
\hline Años de experiencia en la explotación & 62 & $1-54$ & $25,6 \pm 13,8$ & 24 & $1-61$ & $26,0 \pm 18,2$ \\
\hline Escala del rebaño (UGM/UTA total) & 62 & $13-394$ & $91,5 \pm 77,6$ & 24 & $5-221$ & $68,3 \pm 65,1$ \\
\hline \multicolumn{7}{|l|}{ Manejo del ternero } \\
\hline Edad destete (semanas) & 62 & $12-48$ & $22,5 \pm 7$ & & & \\
\hline Prolificidad & 62 & $0,1-1,3$ & $0,56 \pm 0,25$ & 24 & $0,2-1,5$ & $0,7 \pm 0,4$ \\
\hline Productividad cárnica (kg/há) & 57 & $81-885$ & $360,4 \pm 196,1$ & 24 & $40-2440$ & $466,3 \pm 495,9$ \\
\hline Ingresos por UGM (US\$) & 45 & $13-460$ & $127,2 \pm 111,6$ & 6 & $59-533$ & $196,7 \pm 169,7$ \\
\hline UGM/explotación & 62 & $5-90$ & $22,9 \pm 16,7$ & 24 & $1-53$ & $17,8 \pm 14,8$ \\
\hline Ingresos provenientes ganadería (\%) & 51 & $5-100$ & $44,7 \pm 31,7$ & 18 & $5-100$ & $51,1 \pm 36,80$ \\
\hline Principal coste variable alimentación animal (\%) & 61 & $81-99$ & $88,30 \pm 4,6$ & 24 & $33-100$ & $84 \pm 21,2$ \\
\hline Principal coste fijo mano de obra (\%) & 62 & $51-100$ & $83,5 \pm 14,9$ & 24 & $73-100$ & $87,5 \pm 6,1$ \\
\hline Costes MOF por UGM & 62 & $166-1686$ & $735,16 \pm 372$ & 24 & $168-5839$ & $1.373 \pm 1.500$ \\
\hline Costes sin MOF por UGM & 62 & $21-490$ & $151 \pm 122$ & 24 & 22-4494 & $430,54 \pm 993$ \\
\hline
\end{tabular}

UGM= Unidad Ganadera Mayor correspondiente a 450 kilos peso vivo; UTA= Unidad de trabajo agrario correspondiente a jornada de 8 horas diarias; MOF= Mano de obra familiar compuesta por integrantes del grupo familiar. 
na y por la tarde) y cambie sus animales uno por uno a pocos metros de distancia. En ese momento deshierba el lugar que ha consumido el ganado con un machete. Asimismo, por las condiciones del relieve y suelo existen labores que requieren una cantidad de mano de obra que no está disponible en estas explotaciones lo que limita, por tanto, realizar cambios importantes en aspectos productivos como, por ejemplo, el cambio de pastos de ciclo largo a ciclo corto.

El relevo generacional es un problema para estas ganaderías, aunque sobre el $70 \%$ de los ganaderos encuestados en ambas orientaciones productivas mencionó tener familiares, especialmente hijos, que continuarán con la actividad ganadera (tabla III). No obstante, los hijos se encuentran en edad escolar o trabajando en otras actividades no agrícolas y no existe la certeza de que estén dispuestos a trabajar en la ganadería especialmente por las condiciones adversas (tanto climatológicas, de relieve como económicas) donde se realiza la actividad.

La edad media del titular de las explotaciones ganaderas encuestadas es de 54 años para los rebaños de cría y de 50 años para los rebaños de ceba. Los años de experiencia del ganadero oscilan alrededor de los 25 años en ambas orientaciones productivas. La escala del manejo es en promedio de 91,5 y 68,3 UGM/UTA para cría y ceba respectivamente explicada por el sistema de pastoreo que utilizan y por la mano de obra utilizada (tabla IV).

\section{Resultados ECONÓMICOS}

El principal ingreso que tienen estas ganaderías son aquéllos provenientes de la actividad pecuaria. En el caso de la cría, en promedio el 44,7\% de los ingresos proviene de este sistema de manejo. En el caso de la ceba, en promedio el 51,1\% (tabla IV). La productividad cárnica media de los ganaderos de cría es de 360,4 kilos/UGM y de 466,3 kilos/UGM en el caso de la ceba. La variabilidad en ambas orientaciones productivas es alta (tabla IV). Los ingresos medios por UGM de los ganaderos de cría son 127,2 dólares/UGM y de 196,7 dólares/UGM en el caso de la ceba. Los ingresos están directamente relacionados con la productividad cárnica $(\mathrm{p}<0,01)$ (tabla IV). Existen regulaciones de precios por parte del aparato estatal que protegen al ganadero, en la práctica estos precios de sustentación no se cumplen ya sea por falta de fiscalización como de normativas que no se adaptan a la realidad de los sistemas ganaderos amazónicos.

El principal coste variable corresponde a la alimentación animal (88,3\% en el caso de la cría y $84 \%$ en el caso de la ceba). El principal coste fijo corresponde a la mano de obra (83,5\% en el caso de la cría y 87,5\% en el caso de la ceba) (tabla IV). Los costes varían significativamente si se considera la mano de obra familiar. Los costes medios por UGM son de 735 dólares/UGM para el caso de la cría y 1.373 dólares/UGM en el caso de la ceba. La diferencia básicamente se explica por el coste de la UTA que en cada caso ha sido valorizado de acuerdo al número de labores o tareas que implica cada orientación productiva considerando el sistema de manejo. Si no se considera la mano de obra familiar los costes medios disminuyen a 151 dólares/UGM para el caso de la cría y 431 dólares/UGM en el caso de la ceba (tabla IV).

Con todo, los resultados económicos de la actividad para ambas orientaciones productivas son negativos. La persistencia de los ganaderos en estos sistemas de manejo se justifica ya que existen otros ingresos agrarios y extra-agrarios que están sustentando su permanencia en la actividad además de la existencia de producción de autoconsumo destinada a la reproducción de la unidad familiar.

\section{DISCUSIÓN}

El tamaño del rebaño en la provincia de Pastaza es similar al de la Amazonía Ecuatoriana el que oscila en torno a una media de 20 cabezas (INEC, 2013). Este comportamiento ha permanecido en el tiempo respondiendo a las características del manejo ganadero amazónico y a las particularidades del sistema. Un ganadero, con el sistema de pastoreo al sogueo, puede manejar de 25 a 13 cabezas dependiendo de la implantación del forraje (Meunier, 2007 y Grijalva et al., 2004). El tamaño del rebaño en la provincia responde también, a la disponibilidad de alimentación animal, que es una variable crítica a la hora de desarrollar la actividad. El Gramalote es el pastizal que tiene mayor popularidad debido a su adaptación y a su vida útil que es de alrededor de 40 años. No obstante, los ganaderos mencionan que los ciclos de utilización de esta gramínea se están alargando cada vez más, hace veinte años el Gramalote era utilizado a los 6 meses, actualmente está siendo utilizado a los 9-10 meses de crecimiento lo que refleja un deterioro manifiesto de la fertilidad del suelo.

La alimentación animal suministrada a través de piensos también es importante (En promedio 40,3 y $33,3 \%$ respectivamente de los ganaderos de cría y ceba declaran utilizarlo representando sobre el $85 \%$ de sus costes variables). El suministro de estos alimentos carece de una planificación nutricional, por tanto, se pone a disposición de todos los animales por igual sin considerar variables como el potencial de producción, preñez, lactancia. No se utilizan arbustivas, plantas proteicas o leguminosas para complementar la dieta del rebaño. Lo anterior no responde a alternativas de producción sostenibles como lo son los sistemas silvopastoriles recomendados por diversas investigaciones para estos ecosistemas en zonas similares en Colombia (Murgueitio and Ibrahim, 2001 y Murgueitio et al., 2011) o la zona norte de la Amazonía Ecuatoriana (Grijalva et al., 2011; INIAP, 2010; y Grijalva, 2009).

La reproducción se realiza de manera natural. La prolificidad es baja lo que refleja deficiencias en la crianza de hembras de reemplazo que se incorporan tarde a la reproducción, afectando los índices de eficiencia y los costos de producción. En el caso de la ceba, los machos en los rebaños están listos para la venta a edades muy altas (en promedio 48 meses), como consecuencia de retrasos en el crecimiento, lo que se relaciona con alternativas inadecuadas de producción. Estos datos son distintos a los encontrados por Grijalva 
et al. (2004) en la Amazonía norte donde la venta de los machos se realiza entre los 18-24 meses.

La edad media del titular de las explotaciones, tanto para cría y ceba, es similar a la informada por el III Censo Agropecuario (INEC, 2002) (alrededor de 50 años). Esta situación informa del envejecimiento de la población y del bajo interés que tienen los jóvenes para continuar con la actividad, especialmente por considerarla de una complejidad y sacrificio importante, en un ecosistema que tiene muchas dificultades para realizar ganadería.

Los sistemas de manejo existentes se caracterizan por su carácter familiar. Esta es una peculiaridad que comparten estas ganaderías con los sistemas de manejo de la Amazonía Ecuatoriana (Nieto y Caicedo, 2012; Grijalva et al., 2004 y Ramírez, 1996).

El pastoreo es básicamente al sogueo. Las cercas eléctricas son inexistentes respondiendo a la ausencia de pastos de ciclo corto que se adapten al ecosistema debido a los bajos niveles de fertilidad del suelo, pronunciadas pendientes y excesivas lluvias (Bravo, 2014). No existen en Pastaza programas de transferencia tecnológica que asesoren a los ganaderos en cuanto a reconversión de sus pasturas de manera contextualizada al territorio amazónico. Por ejemplo, el uso de cercas para manejar pastos de ciclo corto puede aumentar de manera importante el costo de la mano de obra y dificultar el manejo del ganado si no existe un asesoramiento adecuado para que se realice su utilización de manera eficiente.

La estructura de costes presenta una clara tendencia. El principal coste variable es el pienso y el principal coste fijo es la mano de obra. Sin embargo, estas estimaciones fueron realizadas por Ríos (2014) y no son de conocimiento de los ganaderos, por tanto, difícilmente pueden tomar decisiones estratégicas con respecto a su gestión ganadera.

La comercialización se realiza por peso vivo y preferentemente a través de intermediarios que son los que capturan la mayor parte del excedente en la cadena agroalimentaria. Según Ríos (2014), en la provincia, el precio al productor por kilo de carne en pie es de 1,0 USD. En el Camal el kilo de carne faenada en cuartos es de 2,2 USD y en la tercena de 3,5 USD (carne con hueso al corte). Si se considera que el tercenista adquiere directamente los animales al productor se puede concluir que el diferencial de precio es de 2,5 USD/ kilo. Por tanto, el eslabón que presenta la mayor vulnerabilidad son los productores que están produciendo con estructuras de costes por encima del precio que reciben por kilo de carne en pie. Esta realidad es una constante que ya se ha reportado en el estudio de Vinueza (1992) donde la captura de excedentes de los ganaderos es reducida situándolos en el eslabón más vulnerable de la cadena agroalimentaria.

La principal fortaleza que tienen los ganaderos es su carácter multifuncional y familiar que les permite permanecer en la actividad y tener un margen de maniobra para enfrentar las fluctuaciones del mercado. Las principales debilidades están referidas a problemas de manejo relacionados con aspectos técnicos de reproducción, de alimentación animal, inexistencia de buenas prácticas pecuarias, bajos precios percibidos y rentabilidades económicas negativas. La principal oportunidad es la mejora de la nutrición animal a través de asesorías técnicas que fomenten las asociaciones de pastos que faciliten la fijación de nutrientes (ejemplo: gramíneas más leguminosas). Finalmente, una amenaza importante es la existencia de políticas públicas de fomento pecuario centralizadas que no consideran las limitaciones productivas del territorio amazónico.

\section{CONCLUSIONES}

Los sistemas de producción cárnica bovina se desarrollan en un entorno frágil, en condiciones extremas con un desempeño económico-productivo deficiente para las ganaderías familiares que la practican.

Estos sistemas de manejo ganadero bovino (cría y ceba) se debe mejorar la eficiencia económica-productiva a través un modelo de gestión y ordenamiento territorial que considere las capacidades ecosistémicas del entorno. En este sentido, la localización de los sistemas productivos en superficies aptas para la ganadería (pendientes menores al 30\%) y aplicar la alternativa tecnológica apropiada es de suma relevancia. Actualmente los ganaderos no cuentan con un sistema de acompañamiento estatal que les permita transitar hacia sistemas de producción sostenibles. La evidencia ha mostrado que el sistema más adecuado es el silvopastoril bajo el concepto de sistema de manejo integral donde los árboles interactúan con los animales y las pasturas. El objetivo último es aumentar la productividad del suelo, diversificar la producción y atenuar los efectos climáticos sobre los pastos y ganado.

La continuidad de la actividad actualmente está en riesgo ya que los jóvenes no cuentan con programas de incentivo para permanecer en las explotaciones lo que pone en riesgo la continuidad de la actividad. Este fenómeno puede verse como una oportunidad para desarrollar programas que fomenten el desarrollo de sistemas ganaderos sostenibles donde los jóvenes sean un importante motor de difusión.

Esta investigación requiere que se realice un estudio de la ganadería por pisos climáticos que permita realizar análisis inferenciales que confirmen los hallazgos más relevantes descritos en este diagnóstico con el objetivo de proponer planes de mejora adaptados a la realidad particular del territorio.

\section{AGRADECIMIENTOS Y FINANCIACIÓN}

Esta investigación ha sido financiada por el Ministerio de Ciencia, Innovación y Tecnología SENESCYT del Gobierno Ecuatoriano a través de su proyecto PROMETEO. Especiales agradecimientos a la Universidad Estatal Amazónica por las facilidades, tanto de equipo humano como logístico, que ha permitido que este estudio fuese posible. 


\section{BIBLIOGRAFÍA}

AGROCALIDAD. 2013. Base de datos control ganadero 2013. Agencia Ecuatoriana de aseguramiento de la calidad, provincia de Pastaza. Ecuador.

Barsky, O.; Díaz, E.; Furche, C. y Mizrahi, R. 1982. Políticas agrarias, colonización y desarrollo rural en Ecuador. OEA-CEPLAES. Quito.

Bravo, C. 2014. El enfoque agroecológico y su contribución en el manejo y conservación del recurso suelo. I Congreso de Sociedad en Armonía con la Naturaleza. Universidad Estatal Amazónica. Puyo, Pastaza-Ecuador.

Benítez, D. 2010. Tecnologías sostenibles de producción ganadera en sistemas frágiles y degradados. Editorial Bayamo. Cuba.

Bustamante, T.; Espinoza, M.; Ruiz, L.; Trujillo, J. y Uquillas, J. 1993. Retos de la Amazonía. Ediciones Abya-Ayala. Quito.

Caballero, H. y Anzules, A. 1994. Producción agropecuaria en la Selva Húmeda de la Región Amazónica. IICA-INIAP. Quito.

Descola, P. 1984. Limitaciones ecológicas y sociales del desarrollo de la Amazonía: un estudio de la Amazonía Ecuatoriana. En: Alicia Ibarra Illánes. Población indígena y desarrollo amazónico. Ediciones Abya-Ayala. Quito.

Ehrhart, C. 2000. ¿Quién participa y quién decide? Encontrando un punto de equilibrio entre ciencia y la realidad de la comunidad. En: Rhoades (ed). Tendiendo puentes entre paisajes humanos y naturales. Ediciones Abya-Ayala. Quito.

Grijalva, J.; Arévalo, V. y Wood, Ch. 2004. Expansión y trayectorias de la ganadería en la Amazonía. Estudio en el Valle de Quijos y Piedemonte, en Selva Alta. Instituto Nacional de Investigaciones Agropecuarias (INIAP). Quito.

Grijalva, J. 2009. La agroforestería y desarrollo de la ganadería en la Amazonía Ecuatoriana: Problemas, impactos y oportunidades. Reunión conjunta de redes de producción animal. Santa Cruz. Proyecto AFAMCATIE-INIAP. Joya de los Sáchilas. 8 pp.

Grijalva, J.; Ramos, R. y Vera A. 2011. Pasturas para sistemas silvopastoriles: alternativas para el desarrollo sostenible de la ganadería en la Amazonia Baja del Ecuador. Boletín técnico n 156. Programa Nacional de Forestería del INIAP. Quito.

INEC. 2002. III Censo Nacional Agropecuario 2001. Instituto Nacional de Estadísticas. Quito.

INEC. 2013. Encuesta de superficie y producción agropecuaria continua 2013. Instituto Nacional de Estadísticas. Quito.

INIAP. 2010. Mejoramientoy recuperación de la investigación, soberanía, seguridad alimentaria y desarrollo agropecuario sostenible en la amazonia. Documento de trabajo. INIAP Joya de los Tsáchilas. Orellana.

Leonard, I.; Andino, M. y Uvidia, H. 2014. Recursos forrajeros autóctonos y promisorios para la ganadería en la provincia de Pastaza. Documento de trabajo. Universidad Estatal Amazónica. Puyo.
Meunier, A. 2007. Ganadería en el sur de la Amazonía Ecuatoriana: Motor de la colonización y base de la economía agraria. ¿̇Será capaz de adaptarse a los nuevos retos? En: Mosaico Agrario: Diversidades y antagonismos socio-económicos en el campo ecuatoriano. Instituto de Investigación para el Desarrollo (IRD). Instituto Francés de Estudios Andinos (IFEA). Quito. 318 pp.

Murgueitio, E. and Ibrahim, M. 2001. Agroforestry as a strategy for restructuring livestock production in Latin America. Ponencia presentada en el XVII Congreso Panamericano de Ciencias Veterinarias. Panamá. Septiembre del 2000.

Murgueitio, E. y lbrahim, M. 2004. Ganadería y medio ambiente en América Latina. Conferencia XII Congreso Venezolano de Producción Animal. Maracay. pp. 187-202.

Murgueitio, R.; Calle, Z.; Uribe, F.; Calle, A. and Solorio, B. 2011 . Native trees and shrubs for the productive rehabilitation of cattle ranching lands. Forest Ecol Manag, 261: 1654-1663.

Nieto, C. y Caicedo, C. 2012. Análisis reflexivo sobre el desarrollo agropecuario sostenible en la Amazonia Ecuatoriana. INIAP-EECA. Joya de los Sáchilas. 102 pp.

Pichon, F. 1993. Colonización y deforestación en la frontera agrícola de la Región Amazónica Ecuatoriana. En: Lucy Ruiz M. Amazonía: Escenarios y conflictos. Quito. pp. 337-374.

Ramírez, P. 1996. Diagnóstico del sector agroproductivo de la Región Amazónica Ecuatoriana: Subsector pecuario. ECORAE. Quito. 120 pp.

Ríos, S. 2014. Cadenas de valor pecuarias bovinas en Pastaza. Lógicas de funcionamiento dentro del modelo ganadero ecuatoriano. Documento de trabajo. Universidad Estatal Amazónica. Proyecto Tipificación de ganado bovino. Puyo. 38 pp.

Rudel, T. y Horowitz, B. 1993. La deforestación tropical. Pequeños agricultores y desmonte agrícola en la Amazonia Ecuatoriana. Ediciones Abya- Yala. Quito.

Sierra, R. and Stallings, J. 1998. The dynamics and social organization of tropical deforestation in northwest Ecuador, 1983-1995. Hum Ecol, 26: 135-161.

Vargas, J.C.; Benítez, D.; Torres, V.; Ríos, S.; Soria, S.; Navarrete, H. y Pérez, M. 2014. Tipificación de los sistemas de producción ganaderos en la provincia Pastaza. Informe de resultado del proyecto: Tipificación de los sistemas de producción ganaderos en la provincia Pastaza. Modelo de gestión. Universidad Estatal Amazónica. Puyo. 81 pp.

Vinueza, R. 1992. Los sistemas de comercialización de ganado bovino, leche y queso en los Cantones Pastaza y Palora. MAG-GTZ/ PROFOGAN. Quito.

Wunder, S. 2000. The economics of deforestation: The example of Ecuador. Mac Millan Press. Londres. 